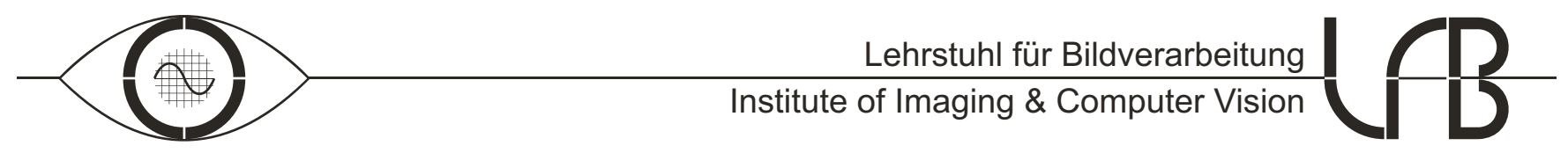

\title{
Halbautomatische Segmentierung von Pulmonalgefaren in CT Daten als Referenz zur Validierung automatischer Verfahren
}

\author{
Jens N. Kaftan and Annemarie Bakai and Florian Maier and Til Aach \\ Institute of Imaging and Computer Vision \\ RWTH Aachen University, 52056 Aachen, Germany \\ tel: +49 24180 27860, fax: +492418022200 \\ web: www.lfb.rwth-aachen.de
}

in: Bildverarbeitung fur die Medizin 2008. See also $\mathrm{BIBT}_{\mathrm{E}} \mathrm{X}$ entry below.

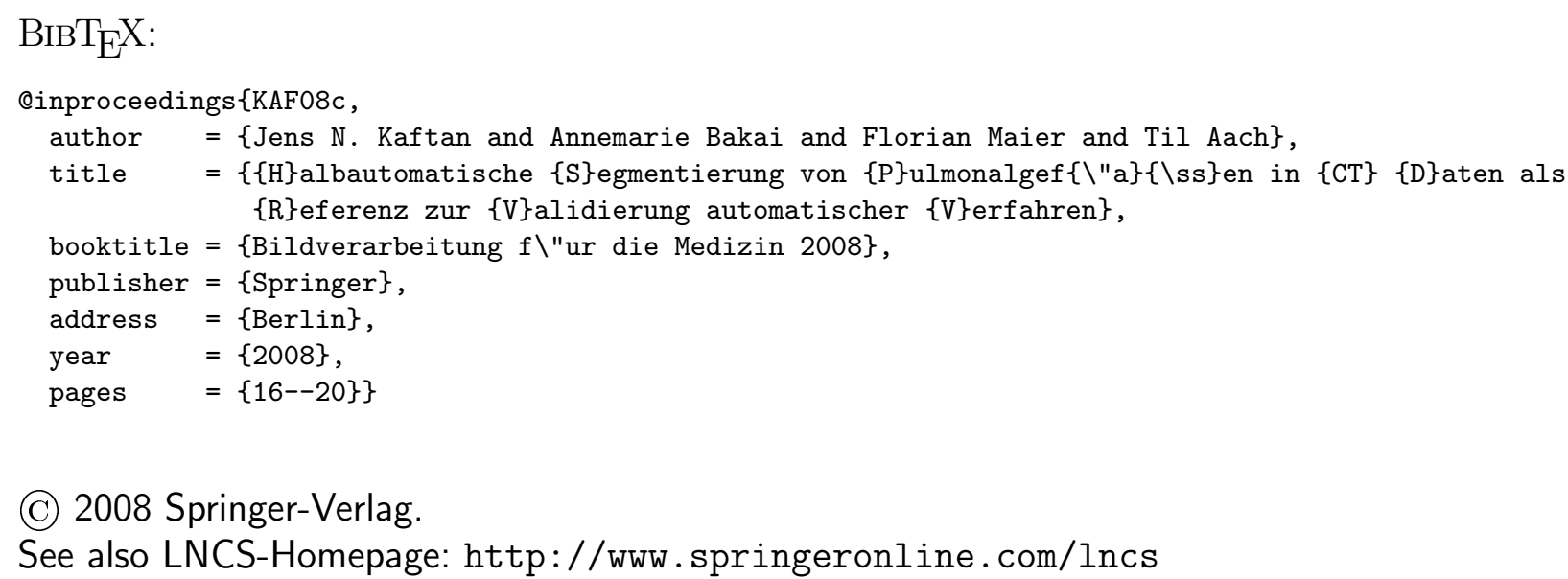





\title{
Halbautomatische Segmentierung von Pulmonalgefäßen in CT Daten als Referenz zur Validierung automatischer Verfahren
}

\author{
Jens N. Kaftan ${ }^{1,2}$, Annemarie Bakai ${ }^{2}$, Florian Maier $^{3}$, Til Aach ${ }^{1}$ \\ ${ }^{1}$ Lehrstuhl für Bildverarbeitung, RWTH Aachen \\ ${ }^{2}$ Siemens Medical Solutions, Computed Tomography Division, Forchheim \\ ${ }^{3}$ Institut für Technische Informatik, Universität Karlsruhe (TH) \\ jens.kaftan@lfb.rwth-aachen.de
}

\begin{abstract}
Kurzfassung. Das Segmentieren von Pulmonalgefäßen in Computertomographie (CT) Daten wurde schon vielfach behandelt und wird z.B. bei der computerunterstützten Detektion von Lungenembolien angewendet. Vielen Segmentierverfahren fehlt jedoch eine quantitative Validierung aufgrund mangelnder Referenzsegmentierungen. Wir stellen ein System zur halbautomatischen Segmentierung von Blutgefäßen in definierten Bereichen der Lunge basierend auf dem Random-Walker-Algorithmus vor. Durch Initialisierung der Methode mittels automatisch generierter Saatpunkte wird die Effizienz des Verfahrens erhöht und die erforderliche Benutzerinteraktion reduziert. Die resultierenden Segmentierungen können zur Validierung von automatischen Verfahren verwendet werden. Exemplarisch evaluieren wir ein vollautomatisches Segmentierverfahren basierend auf dem Fuzzy-Connectedness-Algorithmus.
\end{abstract}

\section{Einleitung}

Pulmonalgefäßsegmentierung in kontrastverstärkten CT Daten (z.B. $[1,2,3]$ ) ist Grundlage für Anwendungen wie z.B. die Detektion von Lungenembolien. Üblicherweise werden solche Segmentierverfahren lediglich qualitativ validiert, da das Erzeugen von manuellen Referenzen aufgrund der Komplexität des Gefäßbaumes schwierig ist. Darüber hinaus kann man die Genauigkeit eines Verfahrens abschätzen, indem man die Anzahl von manuell markierten Mittellinien-Punkten, die von der Segmentierung beinhaltet werden, prozentual bestimmt [1], was aber keinerlei Rückschlüsse auf fehlerhaft positive Regionen zulässt. Wu et al. [2] vergleichen ihre Segmentierergebnisse aus künstlich verrauschten, klinischen Daten mit dem Ergebnis aus den Originaldaten. Damit lässt sich zwar die Robustheit des Verfahrens zeigen, nicht aber die Genauigkeit selbst bewerten.

Wir stellen ein schnelles, halbautomatisches Verfahren zur Erzeugung von Referenzsegmentierungen in definierten Bereichen der Lunge, basierend auf dem Random-Walker-Algorithmus [4,5], vor. Durch Initialisierung des Verfahrens mit effizient generierten Saatpunkten wird die Interaktionszeit soweit reduziert, dass das Verfahren anwendbar für diese ansonsten langwierige Aufgabe wird. Das Ergebnis kann interaktiv so lange verfeinert werden, bis es zufriedenstellend präzise 
ist, wobei jedes beliebige Segmentierergebnis durch Definition ausreichend vieler Saatpunkte erzeugt werden kann. Das Resultat kann dann zur Validierung und Optimierung automatischer Verfahren herangezogen werden.

\section{Material und Methoden}

Halbautomatische Segmentierverfahren erlauben eine schnellere und reproduzierbarere Segmentierung als manuelle Verfahren. Dabei verbleibt die Aufgabe der Detektion (z.B. von Saatpunkten) beim Benutzer, wobei die Delineation vom Computer übernommen wird.

Der Random-Walker-Algorithmus [4,5] ist ein graphbasierter Ansatz zur interaktiven Segmentierung, bei dem die Voxel Knoten entsprechen und banachbarte Voxel durch gewichtete Kanten verbunden sind

$$
w_{i j}=\exp \left(-\beta \cdot d_{i j} \cdot\left|I\left(\mathbf{x}_{i}\right)-I\left(\mathbf{x}_{j}\right)\right|\right)
$$

wobei $I\left(\mathbf{x}_{i}\right)$ der Intensitätswert des Voxels $i$ in HU-Werten, $d_{i j}$ dem Abstand zwischen $i, j$ in mm entsprechen und $\beta$ ein frei wählbarer Parameter ist, der angibt wie stark die Bildkanten berücksichtigt werden sollen (hier: $\beta=150$ ). Unter der Vorraussetzung, dass ein Benutzer einen Teil der Voxel bereits als Vorderund Hintergrund Saatpunkte definiert hat, berechnet er anschaulich gesehen die Wahrscheinlichkeit für jeden verbleibenden Knoten, dass ein sich auf dem Graphen zufällig fortbewegender Spaziergänger zuerst einen Saatpunkt des Vorderoder Hintergrunds erreicht, wobei Kanten mit höherer Gewichtung wahrscheinlicher passiert werden.

Es kann gezeigt werden, dass die gesuchten Wahrscheinlichkeiten sich durch Lösen des diskretisierten Dirichletproblems berechnen lassen. Numerisch kann dies effizient durch das Konjugierte-Gradienten-Verfahren gelöst werden. Um dieses Verfahren anwendbar bezüglich Interaktionsaufwand und Rechenzeit für die Erzeugung von Referenzsegmentierungen der Pulmonalgefäße zu machen, haben wir es um automatische Initialisierungsschritte erweitert.

\section{$2.1 \quad$ Initialisierung}

Um das zu segmentierende Volumen überschaubar zu halten und den Rechenaufwand zu begrenzen, wird zunächst eine $3 D$ Region of Interest (ROI) ausgewählt und automatisch Saatpunkte generiert. Zusätzlich werden Initialisierungswahrscheinlichkeiten abhängig vom Intensitätswert jedes Voxels berechnet um die Konvergenzgeschwindigkeit des numerischen Verfahrens zu erhöhen. Um eine sinnvolle Wahrscheinlichkeitsfunktion aufzustellen haben wir verschiedene, mit diesem Verfahren segmentierte ROIs unterschiedlicher Patienten bezüglich ihrer Intensitätsverteilung untersucht (Abb. 1a). Unter der Annahme, dass die Intensitätswerte $I(\mathbf{x})$ einer Verteilung $G$ entspricht, die sich aus zwei Gauss-Verteilungen $G_{V G}$ und $G_{H G}$ für Blutgefäße (Vordergrund) und NichtBlutgefäße (Hintergrund) zusammensetzt, können die Parameter $\mu, \sigma$ der bedingten Wahrscheinlichkeitsdichtefunktion geschätzt werden. Daraus lässt sich 
eine Wahrscheinlichkeitsverteilung definieren, in der die Hintergrundwahrscheinlichkeit $P(H G \mid \mathbf{x})=1$ für $I(\mathbf{x}) \leq \mu_{H G}$ und die Vordergrundwahrscheinlichkeit $P(V G \mid \mathbf{x})=1$ für $I(\mathbf{x}) \geq \mu_{V G}$ ist (Abb. 1b). Alle Voxel, die eines dieser Kriterien erfüllen werden als Vorder- bzw. Hintergrundsaatpunkte definiert. Dazwischen fällt die Wahrscheinlichkeit entsprechend einer Gaussfunktion mit den bestimmten Parametern $\mu, \sigma$ ab, wobei Vorder- und Hintergrundwahrscheinlichkeit komplementär sein müssen. Zusätzlich erlauben wir einen Bereich, für den keine der beiden Klassen bevorzugt wird: $P(V G \mid \mathbf{x})=P(H G \mid \mathbf{x})=0.5$.

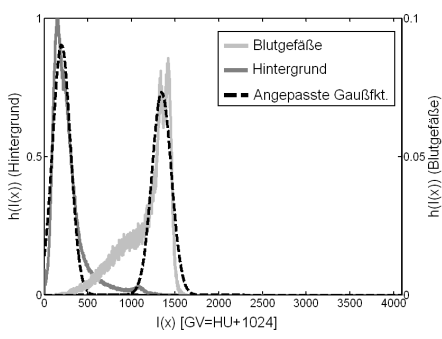

(a) Histogramm

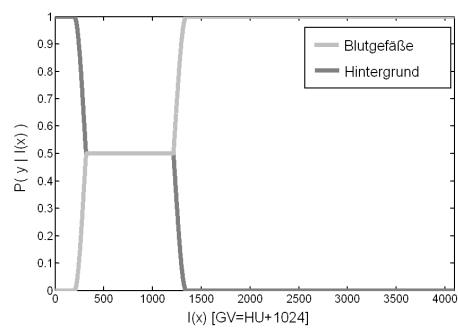

(b) Wahrscheinlichkeitsverteilung

Abb. 1. (a) Histogramm über verschiedene ROIs getrennt nach Blutgefäße (hellgrau) und Hintergrund (dunkelgrau) und die entsprechenden angepassten Gaußfunktionen mit $\mu_{H G}=-826 \mathrm{HU}, \sigma_{H G}=102 \mathrm{HU}$ und $\mu_{V G}=322 \mathrm{HU}, \sigma_{V G}=111 \mathrm{HU}$. Man beachte die unterschiedliche Achsenskalierung für beide Klassen. (b) Wahrscheinlichkeitsverteilung zur Initialisierung des Random-Walker-Algorithmus.

\subsection{Anwendung}

Die mit dem vorgestellten Verfahren erzeugten Segmentierungen können vom Benutzer so lange verfeinert werden, bis das Resultat qualitativ als Referenz genügt und damit zur Validierung automatischer Verfahren herangezogen werden kann. Exemplarisch zeigen wir eine quantitative Validierung für ein automatisches Verfahren [3]. Zur automatischen Segmentierung werden zunächst Blutgefäßregionen mit sehr hoher Spezifität schwellwertbasiert detektiert und jeder resultierende, zusammenhängende Teilbaum wird zu einem oder mehreren Saatpunkten reduziert. Anschließend wird ein Fuzzy-Connectedness-Regionenwachstum [6] gestartet, dass jedem Voxel ein wahrscheinlichkeits-ähnliches Maß zuweist, welches angibt wie stark ein Voxel mit einem der Saatpunkte verbunden ist und damit zum Blutgefäßbaum gehört. Die Zusammengehörigkeit benachbarter Voxel $i, j$ berechnet sich mittels einer intensitätsbasierten Wahrscheinlichkeitsfunktion:

$$
g\left(I\left(\mathbf{x}_{i}\right), I\left(\mathbf{x}_{j}\right)\right)=\exp \left(-\frac{1}{2 \sigma^{2}}\left(\frac{I\left(\mathbf{x}_{i}\right)-I\left(\mathbf{x}_{j}\right)}{2}-\mu\right)^{2}\right)
$$

wobei $\mu$ hier dem mittleren HU-Wert der Saatpunkte entspricht und $\sigma$ frei wählbar ist (hier: $\sigma=250$ ). Das Wachstum wird abgebrochen, wenn die Wahrscheinlichkeit unter eine bestimmte Schwelle sinkt. Abschließend erhält man eine binäre Segmentierung durch Anwendung eines Schwellwerts auf die Wahrscheinlichkeitskarte, welcher die Sensitivität/Spezifität des Verfahrens beeinflusst. 


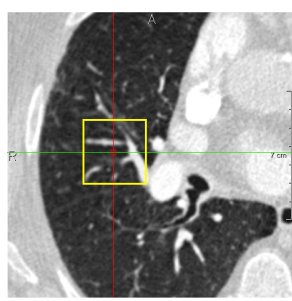

(a)

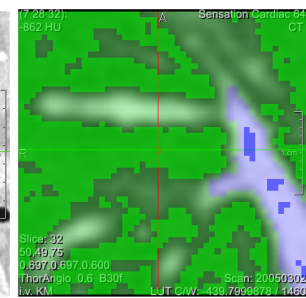

(b)

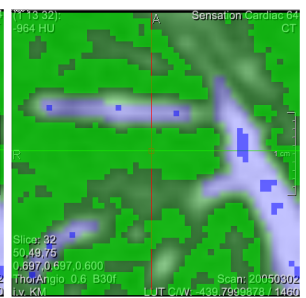

(c)

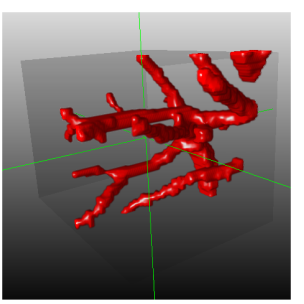

(d)

Abb. 2. (a) Lungenausschnitt mit ROI (gelb). (b) Initiale Segmentierung ohne manuelle Nachverarbeitung. Vorder- und Hintergrundsaatpunkte sind opak blau bzw. grün dargestellt, während die resultierende Segmentierung halbtransparent gezeigt wird. (c) Segmentierung nach Hinzufügen weiterer Saatpunkte. (d) 3D Darstellung.

\section{Ergebnisse}

Das vorgestellte Verfahren erlaubt eine schnelle und präzise halbautomatische Segmentierung der Blutgefäße innerhalb der ROI. Das Segmentierergebnis ist exemplarisch in Abbildung 2 dargestellt, basierend lediglich auf der automatischen Initialisierung und einer Random-Walker-Anwendung (b) und nach weiterer Benutzerinteraktion (c-d). Während die automatische Detektion von Saatpunkten den Interaktionsaufwand erheblich reduziert, bewirkt die Vor-Initialisierung mit der Wahrscheinlichkeitsfunktion aus Abb. 1b lediglich eine Zeitersparnis von maximal 4-5\% für die erste Random-Walker Berechnung.

Mit dem somit zur Verfügung stehenden Verfahren können Segmentierergebnisse im Detail bezüglich ihrer Fehler (fehlerhaft positiv/negativ segmentierte Voxel) untersucht werden (Abb. 3b), aus denen Validierungsmaße, wie z.B. Sensitivität/Spezifität (Abb. 3c) berechnet werden können. Man kann sehen, dass das automatische Verfahren eine Sensitivität (Anteil korrekt positiv segmentierter Voxel) von $80 \%$ bei einer Spezifität (Anteil korrekt negativ segmentierter Voxel) von $95 \%$ erreicht.

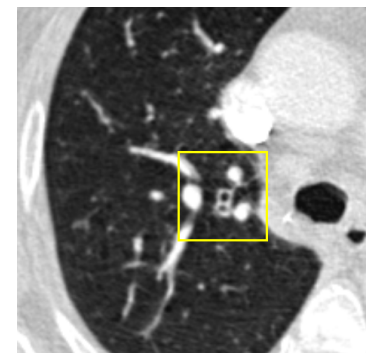

(a)

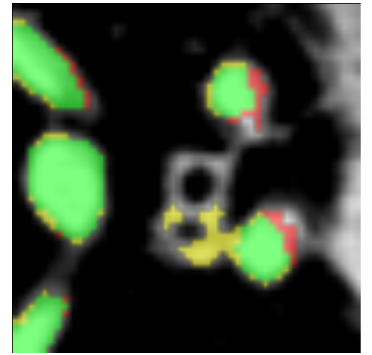

(b)

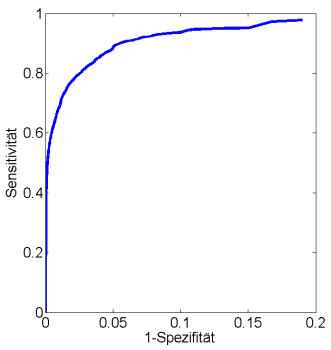

(c)

Abb. 3. (a) ROI. (b) Vergleich automatischer mit Referenzsegmentierung. Korrekt positive Regionen sind grün, fehlerhaft positive gelb und fehlerhaft negative rot dargestellt. (d) ROC Kurve über 5 Patienten mit je 3 ROIs der Größe $50^{3}$ Voxel. 


\section{Diskussion}

Wir haben ein halbautomatisches Verfahren zur Erzeugung von Referenzsegmentierungen des Blutgefäßbaumes in definierten Bereichen der Lunge vorgestellt. Wir nutzen dabei den Random-Walker-Algorithmus, den wir um automatische Initialisierungsschritte erweitert haben, wodurch das Verfahren anwendbar für diese ansonsten langwierige Aufgabe wird.

Streng genommen müsste die Qualität der so erzeugten Referenzsegmentierungen ebenfalls untersucht werden, was aus Mangel an einem exakten Goldstandard für klinische Daten wiederum nicht möglich ist. Das vorgestellte Verfahren weist jedoch einige Merkmale auf, die darauf hindeuten, dass die so erzeugten Segmentierungen zur Validierung genutzt werden können:

- Mit dem Random-Walker-Algorithmus lässt sich jede beliebige Segmentierung durch Definition ausreichend vieler Saatpunkte erzeugen [5].

- Es wird nur eine kleine Zahl von Saatpunkten benötigt um eine Segmentierung zu erzeugen, die einer Manuellen nahezu identisch ist [4].

- Änderungen an der Anordnung der Saatpunkte resultieren nur in einer kleinen Änderung des Segmentierergebnisses [4], wodurch das Verfahren reproduzierbarere Ergebnisse liefert als manuelle Methoden.

Aufgrund der Automatisierung beider Teilschritte - Saatpunktgewinnung und Strukturdelineation - kann ein präzises Segmentierergebnis mit akzeptablem Zeitaufwand erzielt werden, welches als Referenz zur Optimierung und Validierung automatischer Verfahren genutzt werden kann. Durch Wahl mehrerer Referenzregionen pro Lunge, randomisiert oder systematisch in verschiedenen Bereichen, kann die Relevanz der Vergleichskriterien weiter erhöht werden.

\section{Literaturverzeichnis}

1. Zhou C, Chan HP, Hadjiiski LM, Patel S, Cascade PN, Sahiner B, et al. Automatic pulmonary vessel segmentation in $3 \mathrm{D}$ computed tomographic pulmonary angiographic (CTPA) images. In: SPIE Medical Imaging. vol. 6144; 2006. p. 1524-1530.

2. Wu C, Agam G, Roy AS, Armato SG III. Regulated morphology approach to fuzzy shape analysis with application to blood vessel extraction in thoracic CT scans. In: SPIE Medical Imaging. vol. 5370; 2004. p. 1262-1270.

3. Kaftan JN, Kiraly AP, Bakai A, Das M, Novak CL, Aach T. Fuzzy Pulmonary Vessel Segmentation in Contrast Enhanced CT Data. In: SPIE Medical Imaging. vol. $6914 ; 2008$. To appear.

4. Grady L, Schiwietz T, Aharon S, Westermann R. Random Walks for Interactive Organ Segmentation in Two and Three Dimensions: Implementation and Validation. In: MICCAI. No. 2 in LNCS 3750; 2005. p. 773-780.

5. Grady L. Random Walks for Image Segmentation. IEEE Transactions on Pattern Analysis and Machine Intelligence. 2006;28(11):1768-1783.

6. Udupa JK, Samarasekera S. Fuzzy Connectedness and Object Definition: Theory, Algorithms, and Applications in Image Segmentation. Graph Models Image Process. 1996;58(3):246-261. 